\title{
Comparative short-term outcomes of enhanced recovery after surgery (ERAS) program and non-ERAS traditional care in elderly patients undergoing lumbar arthrodesis: a retrospective study
}

Zhong-En Li $i^{1,2,3}$, Shi-Bao Lu ${ }^{1,2^{*}}$, Chao Kong ${ }^{1,2,3}$, Wen-Zhi Sun ${ }^{1,2,3}$, Peng Wang ${ }^{1,2,3}$ and Si-Tao Zhang ${ }^{1,2}$

\begin{abstract}
Background: Enhanced recovery after surgery (ERAS) program is an evidence-based improvement over non-ERAS traditional care. The aim of the present study was to analyze the safety, feasibility, and efficacy of an ERAS program in patients over 70 years undergoing lumbar arthrodesis by comparison with non-ERAS traditional care.

Methods: During January 2018 to December 2018, patients enrolled received non-ERAS traditional care, while the ERAS program was implemented from January to December 2019. Demographic characteristics, comorbidities, surgical data and postoperative recovery parameters were collected from all patients. Postoperative pain scores were evaluated by visual analog scales (VAS). The clinical outcomes were length of stay (LOS), postoperative complications and postoperative pain scores. Compliance results were also collected.

Result: A total of 127 patients were enrolled, including 67 patients in the non-ERAS traditional care group and 60 patients in the ERAS group. The demographic characteristics and comorbidities of the two groups showed no significant differences. The LOS of patients treated with ERAS program (13.6 \pm 4.0 days) was significantly less than that of patients treated with non-ERAS traditional care $(15.6 \pm 3.9$ days $)(p=0.034)$. Complication rate was $8.3 \%$ in the ERAS group versus $20.9 \%$ in the non-ERAS traditional care group $(p=0.048)$. VAS (back) in the ERAS group was significantly lower on postoperative day (POD) 1 and POD2. Postoperative recovery parameters were improved in the ERAS group. The overall compliance with the ERAS program was $94 \%$.
\end{abstract}

Conclusions: Based on our results, ERAS program is safer and more effective for elderly patients over 70 undergoing lumbar arthrodesis than non-ERAS traditional care.

Keywords: Enhanced recovery after surgery, Elderly patients, Lumbar arthrodesis, Length of stay, Complication

\footnotetext{
* Correspondence: xuanwuspine@sina.com

'Department of Orthopedics, Xuanwu Hospital Capital Medical University, Beijing, China

${ }^{2}$ National Clinical Research Center for Geriatric Diseases, Beijing, China

Full list of author information is available at the end of the article
}

(c) The Author(s). 2021 Open Access This article is licensed under a Creative Commons Attribution 4.0 International License, which permits use, sharing, adaptation, distribution and reproduction in any medium or format, as long as you give appropriate credit to the original author(s) and the source, provide a link to the Creative Commons licence, and indicate if changes were made. The images or other third party material in this article are included in the article's Creative Commons licence, unless indicated otherwise in a credit line to the material. If material is not included in the article's Creative Commons licence and your intended use is not permitted by statutory regulation or exceeds the permitted use, you will need to obtain permission directly from the copyright holder. To view a copy of this licence, visit http://creativecommons.org/licenses/by/4.0/. The Creative Commons Public Domain Dedication waiver (http://creativecommons.org/publicdomain/zero/1.0/) applies to the data made available in this article, unless otherwise stated in a credit line to the data. 


\section{Background}

With the advent of an aging society, the number of elderly patients with lumbar degenerative diseases requiring surgery is increasing. Lumbar arthrodesis is the most common surgical method to treat lumbar degenerative diseases [1-3]. Due to the decline of physical reserve capacity and increased comorbidities in elderly patients, the risks of perioperative complications and prolonged hospitalization need to be actively managed in clinical practice.

Enhanced recovery after surgery (ERAS) program is an evidence-based improvement over non-ERAS traditional care. Individual ERAS items are implemented during the patient's journey through the pre-operative, intraoperative, and post-operative phases of care. Moreover, these combined factors are associated with a major shift in clinical routines, from old practices to new standardized patient care pathways $[4,5]$. The ERAS program can reduce organ dysfunction and surgical stress, thereby reducing postoperative complications and length of stay (LOS) [6].

Notably, the ERAS program was first proposed based on a study in patients undergoing colon surgery. Elderly patients were the target group selected at the beginning of the program design, and the postoperative LOS was shortened to 2-3 days in patients over 80 years of age [7]. As the ERAS protocols were adopted in other surgical specialties, many factors including the characteristics of the perioperative period, the stress on the patients, and compliance rate may affect the benefit of ERAS [811]. Kehlet et al. recently emphasized that the ERAS program should be designed and evaluated according to specific surgery and population [12, 13]. Though the ERAS program for spinal surgery has received some attention, most of the current studies focused on minimally invasive surgery [14-18]. There are scarce data on the implementation of ERAS program in open lumbar surgery, especially in elderly patients.

The aim of the present study was to analyze the safety, feasibility, and efficacy of an ERAS program in patients over 70 years undergoing lumbar arthrodesis by comparison with non-ERAS traditional care.

\section{Methods}

\section{Patients}

This retrospective case-review study was conducted from January 2018 to December 2019 after the approval of the Hospital Committee and Institutional Review Board (permit data January 2018; no. 2018007) and results were reported in accordance to the STROBE statements [19]. During the first year of this study, patients enrolled received non-ERAS traditional care, while the ERAS program was implemented from January to December 2019. We prospectively collected perioperative data with analysis for the ERAS patients from medical records and database. All patients provided informed consent to the work.

The inclusion criteria included patients older than 70 years who underwent open lumbar arthrodesis with pedicle screw fixation and the main diagnosis was lumbar stenosis. Surgical treatment was performed at the following indications: (1) no improvement in clinical manifestations despite conservative treatment for a minimum of 6 months, where conservative treatment included medication, physical therapy, and up to 3 injection treatments; and (2) any pathologies requiring fusion procedure following decompression, which were degenerative spondylolisthesis, isthmic spondylolisthesis, and foraminal stenosis in the study. The exclusion criteria included (1) circumferential fusion (a combination of anterior and posterior surgery); (2) a history of previous lumbar spine surgery.

All patients underwent a standard midline posterior lumbar decompression and interbody fusion was preferred in the decompressed levels. All surgeries were performed by the same surgeon and the ERAS program was performed by the same group of trained anesthesiologists and nurses.

\section{Components of non-ERAS traditional care program and ERAS program}

Our ERAS program was designed based on previously published pathways and the differences of details between the non-ERAS traditional care group and the ERAS group were compared in Table $1[8,11,20,21]$. Non-ERAS traditional care program was performed with no unified guidelines.

The perioperative multimodal analgesia (MMA) was the combination of different pain signaling pathways to improve pain control and reduce opioids.

We assigned dedicated stuff who did not participate in the study to record compliance.

\section{Data collection}

Demographic characteristics including age, gender, body mass index (BMI), surgical level, smoker, comorbidities, American Society of Anesthesiologists (ASA) classification and preoperative Oswestry Disability Index (ODI) were collected from all patients. Surgical data including LOS, surgical time, estimated blood loss, complications and 30-day hospital readmission were recorded. Leg and back pain scores were evaluated by visual analog scales (VAS). We also recorded postoperative recovery parameters, including early ambulation, early oral feeding or early removal of catheter. The number of patients who received nutrition intervention after nutrition screening was documented. LOS was defined as the time from admission to discharge recorded in the medical record. 


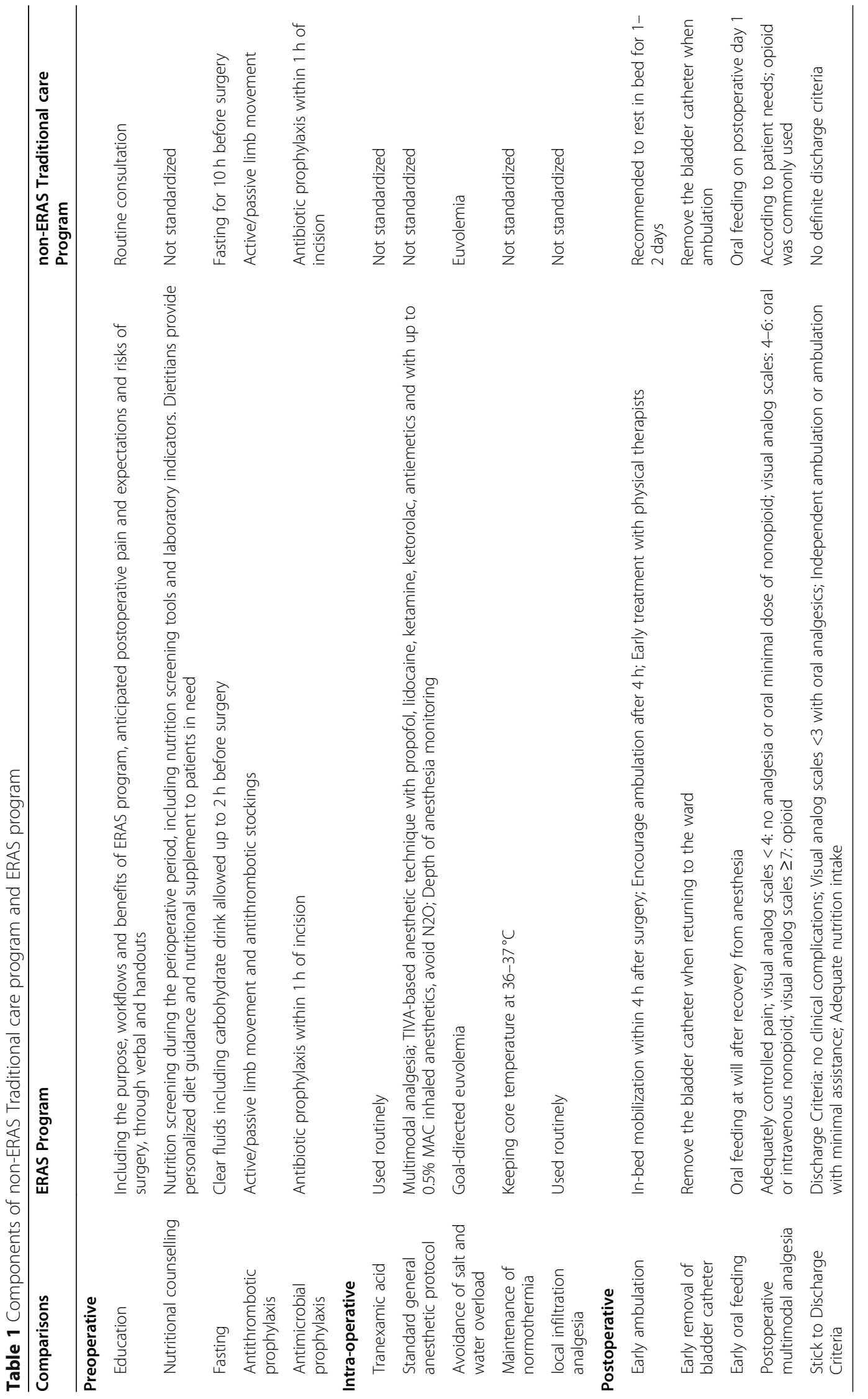


Complications referred to all postoperative complications that occurred within 30 days after surgery. All of the complications were determined by outpatient or follow-up doctors.

\section{Statistics}

Continuous variables were expressed as mean value \pm standard deviation (Mean $\pm \mathrm{SD}$ ), and they were compared using student $t$ test when parametric assumptions were met, otherwise, the Mann-Whitney test was performed. Statistical analysis for categorical variable was performed by the Chi-square test. Multivariate Logistic regression was used to determine association of risk factors with postoperative complications. Multivariable linear regression analysis was used to determine the association of risk factors with LOS. Differences and regressions were considered significant with $\mathrm{p}<0.05$. Statistical analysis was performed using SPSS version 16.0 for Windows (SPSS Inc., Chicago, IL, USA).

\section{Results}

Patient demographics

A total of 127 patients were enrolled, including $67 \mathrm{pa}$ tients in the non-ERAS traditional care group and $60 \mathrm{pa}$ tients in the ERAS group. The demographic characteristics are detailed in Table 2. There were no statistical differences in age $(p=0.430)$, gender $(p=$ $0.675)$, BMI $(p=0.769)$, surgical level $(p=0.961)$ between the two groups of patients. Comorbidities, ASA classification $(p=0.647)$ and preoperative ODI $(p=0.419)$ of the two groups also showed no significant differences. (Table 2).

\section{Clinical outcomes}

The LOS of patients treated with the ERAS program (13.6 \pm 4.0 days, range from 7 to 20 days) was significantly shorter than that of patients treated with nonERAS traditional care $(15.6 \pm 3.9$ days, range from 7 to 24 days). The surgical time in the ERAS group (176.6 \pm $56.7 \mathrm{~min})$ was similar to the non-ERAS traditional care group $(190.4 \pm 89.3 \mathrm{~min}) \quad(p=0.289)$. Estimated blood

Table 2 Demographic Characteristics of Patients

\begin{tabular}{|c|c|c|c|}
\hline & ERAS & non-ERAS Traditional care & $P$ value \\
\hline Patients (n) & 60 & 67 & \\
\hline Age (range) & $73.6 \pm 3.2(70-84)$ & $74.3 \pm 4.2(70-85)$ & 0.430 \\
\hline Gender (n) & & & 0.675 \\
\hline Male & 22 & 27 & \\
\hline Females & 38 & 40 & \\
\hline $\mathrm{BMI}$ & $25.9 \pm 3.85$ & $26.0 \pm 4.08$ & 0.769 \\
\hline Surgical level & & & 0.961 \\
\hline 1-2levels & 45 & 50 & \\
\hline$\geq 3$ levels & 15 & 17 & \\
\hline Smoker (n) & 7 & 8 & 0.962 \\
\hline \multicolumn{4}{|l|}{ Comorbidities (n) } \\
\hline Hypertension & 32 & 36 & 0.964 \\
\hline Diabetes & 19 & 20 & 0.825 \\
\hline Ischemic heart disease & 5 & 8 & 0.503 \\
\hline Stroke & 3 & 2 & 0.560 \\
\hline Arrhythmias & 5 & 5 & 0.856 \\
\hline Gastrointestinal & 2 & 1 & 0.495 \\
\hline Chronic lung disease & 3 & 2 & 0.560 \\
\hline Parkinson disease & 1 & 2 & 0.625 \\
\hline Depression & 2 & 4 & 0.911 \\
\hline ASA classification & & & 0.647 \\
\hline 2 & 31 & 40 & \\
\hline 3 & 23 & 22 & \\
\hline 4 & 6 & 5 & \\
\hline Preoperative ODI (\%) & $59.3 \pm 14.3$ & $61.6 \pm 14.7$ & 0.419 \\
\hline
\end{tabular}


loss in the ERAS group $(365.6 \pm 207.4 \mathrm{ml})$ was similar to the non-ERAS traditional care group $(337.5 \pm 194.9 \mathrm{ml})$ $(p=0.118)$. Complication rate was $8.3 \%$ in the ERAS group versus $20.9 \%$ in the non-ERAS traditional care group $(p=0.048)$. After implementing ERAS program, the proportion of early ambulation increased from 7.5 to $70 \%$. The proportion of patients with early oral feeding and removal of catheter increased respectively from 3 to $86.7 \%$ and from 14.9 to $80 \%$. By comparison, more patients received nutrition interventions (19.4 to $45 \%$ ). (Table 3).

Complications are summarized in Table 4. Among the 5 patients with complications in the ERAS group, two had superficial infection, one suffered from cerebrospinal fluid leakage, one suffered from electrolyte abnormality and one experienced arrhythmia. Complications in the non-ERAS traditional care group were distributed as follows: urinary tract infection, partial root injuries, arrhythmia, deep vein thrombosis and deep wound infection, each in one case, two patients suffered from superficial infection, three patients suffered from electrolyte abnormality, and four patients experienced delirium. There were no complications requiring readmission within 30 days of surgery in both groups.

Table 3 Clinical outcomes

\begin{tabular}{|c|c|c|c|}
\hline & $\begin{array}{l}\text { ERAS } \\
(n=60)\end{array}$ & $\begin{array}{l}\text { non-ERAS Traditional } \\
\text { care }(n=67)\end{array}$ & $P$ value \\
\hline LOS (range), days & $\begin{array}{l}13.6 \pm 4.0 \\
(7-20)\end{array}$ & $15.6 \pm 3.9(7-24)$ & 0.034 \\
\hline Surgical time, min & $186.6 \pm 56.7$ & $190.4 \pm 89.3$ & 0.289 \\
\hline $\begin{array}{l}\text { Estimated blood } \\
\text { loss, ml }\end{array}$ & $337.5 \pm 194.9$ & $365.6 \pm 207.4$ & 0.118 \\
\hline $\begin{array}{l}\text { Complications, } \\
\text { n (rate) }\end{array}$ & & & 0.048 \\
\hline No & $55(91.7)$ & $53(79.1)$ & \\
\hline Yes & $5(8.3)$ & $14(20.9)$ & \\
\hline $\begin{array}{l}\text { Early ambulation, } \\
\mathrm{n} \text { (rate) }\end{array}$ & & & $<0.0001$ \\
\hline No & $18(30.0)$ & $62(92.5)$ & \\
\hline Yes & $42(70.0)$ & $5(7.5)$ & \\
\hline $\begin{array}{l}\text { Early oral feeding, } \\
\mathrm{n} \text { (rate) }\end{array}$ & & & $<0.0001$ \\
\hline No & $8(13.3)$ & $65(97.0)$ & \\
\hline Yes & $52(86.7)$ & $2(3.0)$ & \\
\hline $\begin{array}{l}\text { Early removal of } \\
\text { catheter, } \mathrm{n} \text { (rate) }\end{array}$ & & & $<0.0001$ \\
\hline No & $12(20.0)$ & $57(85.1)$ & \\
\hline Yes & $48(80.0)$ & $10(14.9)$ & \\
\hline $\begin{array}{l}\text { Nutritional intervention, } \\
\mathrm{n} \text { (rate) }\end{array}$ & & & 0.002 \\
\hline No & $33(55.0)$ & $54(80.6)$ & \\
\hline Yes & $27(45.0)$ & 13 (19.4) & \\
\hline
\end{tabular}

Table 4 List of complications of the two groups

\begin{tabular}{lll}
\hline Complications & ERAS & Non-ERAS traditional care \\
\hline Total & 5 & 14 \\
Superficial infection & 2 & 2 \\
Electrolyte abnormality & 1 & 3 \\
Arrhythmia & 1 & 1 \\
Urinary tract infection & 0 & 1 \\
Deep vein thrombosis & 0 & 1 \\
Deep wound infection & 0 & 1 \\
Delirium & 0 & 4 \\
Surgical complications & & \\
$\quad$ Cerebrospinal fluid leakage & 1 & 0 \\
Partial root injuries & 0 & 1 \\
\hline
\end{tabular}

Average VAS (back) in the ERAS group was significantly lower than that in the non-ERAS traditional care group on postoperative day (POD) 1 (3.8 \pm 1.7 versus $5.7 \pm 2.3, p=0.028)$ and POD2( $3.6 \pm 1.9$ versus $4.5 \pm 2.2$, $p=0.043)$. Whereas, there were no statistically significant differences between the groups on POD3 (3.1 \pm 1.2 versus $3.8 \pm 1.7, p=0.122)$ and POD4 $(2.7 \pm 0.5$ versus $3.2 \pm 0.9, p=0.363)$. There were no differences in the VAS (leg) postoperatively. (Table 5).

\section{Multivariable analyses}

Multivariable linear regression analysis was performed to determine the association of various factors with LOS. Implementation of the non-ERAS traditional care program $(p=0.006)$ and higher preoperative ODI $(p=$ 0.012 ) were correlated with prolonged LOS. On the other hand, age $(p=0.579)$, BMI $(p=0.351)$, surgical level $\geq 3 \quad(p=0.083)$ and surgical time $(p=0.127)$ were not related to LOS (Table 6).

Multivariable logistic regression showed that implementation of ERAS program $(p=0.040)$ was associated with decrease in complications. The other characteristics were not associated with complications. (Table 7).

\section{Compliance with the ERAS program}

Compliance with the ERAS program is illustrated in Table 8. In general, high compliance rates were achieved for the pre-operative and Intra-operative ERAS items. In contrast, compliance of the postoperative ERAS items was relatively low: early ambulation (70\%), early removal of bladder catheter (86.7\%), early oral feeding (80\%), stick to discharge criteria (78.3\%). The overall compliance was $94 \%$.

\section{Discussion}

To the best of our knowledge, this is the first study focusing on the implementation of ERAS program in elderly patients undergoing lumbar arthrodesis. Similar to 
Table 5 The comparisons of average VAS between the two groups on POD1-4

\begin{tabular}{|c|c|c|c|c|c|c|}
\hline \multicolumn{4}{|l|}{ VAS (back) } & \multicolumn{3}{|l|}{ VAS (leg) } \\
\hline comparison & ERAS & non-ERAS Traditional care & $P$ value & ERAS & non-ERAS Traditional care & $P$ value \\
\hline POD 1 & $3.8 \pm 1.7$ & $5.7 \pm 2.3$ & $p=0.028$ & $3.2 \pm 1.5$ & $3.8 \pm 1.7$ & $p=0.137$ \\
\hline POD 2 & $3.6 \pm 1.9$ & $4.5 \pm 2.2$ & $p=0.043$ & $2.5 \pm 1.6$ & $3.5 \pm 1.2$ & $p=0.088$ \\
\hline POD 3 & $3.1 \pm 1.2$ & $3.8 \pm 1.7$ & $p=0.122$ & $2.0 \pm 1.3$ & $2.5 \pm 0.9$ & $p=0.230$ \\
\hline POD 4 & $2.7 \pm 0.5$ & $3.2 \pm 0.9$ & $p=0.363$ & $1.8 \pm 0.8$ & $2.0 \pm 1.0$ & $p=0.594$ \\
\hline
\end{tabular}

the results of ERAS program in minimal invasive lumbar surgery $[15,16]$, this study found that the ERAS program significantly reduced the incidence of complications and LOS, and the majority of elderly patients could complete the pathway. The standardized multimodal analgesia significantly reduced postoperative pain levels in the ERAS group. Only patients undergoing lumbar arthrodesis were included in this study to avoid the bias caused by surgical types.

Due to the influence of non-clinical factors such as culture, doctor-patient relationship, and insurance system, although the LOS of patients in the ERAS group was significantly shortened in our study, it was still longer than that in other studies $[5,17,18]$. It is reported that elderly patients undergoing lumbar spine surgery have extended LOS, because patients with advanced age are more likely to suffered from more baseline comorbidities and postoperative complications that may require further medical or surgical intervention [22, 23]. Moreover, elderly patients commonly experienced issues of decreased ability to perform daily activities and difficulty with self-care, which leads to reluctance to discharge, even if the criteria are met [24]. In our study, the LOS was prolonged to 24 days of 1 patient in the nonERAS traditional care group because of deep wound infection. The patient had to be taken to the operating room for wound washout. Another patient in the nonERAS traditional care group delayed LOS to 22 days because of postoperative delirium. In our ERAS group, 1 patient experienced delayed discharge (LOS $=20$ days) for nonsurgical superficial infection. Our study also showed that higher preoperative ODI was associated

Table 6 Multivariable linear regression for LOS

\begin{tabular}{lll}
\hline Characteristic & \multicolumn{2}{l}{ Multivariable linear regression for LOS } \\
\cline { 2 - 3 } & Coefficient $(\mathbf{9 5} \% \mathrm{Cl})$ & $\boldsymbol{p}$ value \\
\hline Age & $0.15(-0.09$ to 0.32$)$ & 0.579 \\
BMI & $-0.37(-0.90$ to 1.12$)$ & 0.351 \\
Surgical level $\geq 3$ & $1.94(-0.76$ to 3.16$)$ & 0.083 \\
ASA $\geq 3$ & $1.15(-0.42$ to 2.53$)$ & 0.238 \\
Surgical time & $2.27(-1.2$ to 3.34$)$ & 0.127 \\
ERAS & $-3.08(-5.12$ to -1.14$)$ & 0.006 \\
Preoperative ODI (\%) & $0.94(0.25$ to 2.04$)$ & 0.012 \\
\hline
\end{tabular}

with prolonged LOS, because worse preoperative motor capacity usually leads to longer time for first ambulation. The implementation of ERAS can reduce occurrence of complications, and provide adequate pain control, which are important components of our discharge criteria.

Similar to previous studies [25-27], our results showed that the implementation of ERAS program was associated with lower complications. We believe that there are several factors in the study contributing to the decrease in complications. Early removal of bladder catheter and standard antimicrobial prophylaxis reduce the risk of infectious complications such as urinary tract infection and wound infection [28-30]. Thrombosis-related complications can be decreased by active/passive limb movement, antithrombotic stockings and early ambulation. Notably, advanced age is one of the main risk factors for postoperative delirium, and the incidence of postoperative delirium after elective lumbar surgery can be as high as $15 \%$, which can lead to nursing difficulties and lower compliance with the ERAS protocols [31-33]. Postoperative delirium has rarely been discussed in ERAS studies, but it is extremely important for the prognosis of patients, especially in elderly patients [12, 34]. In this study, no patients in the ERAS group suffered from postoperative delirium in contrast to 4 cases in nonERAS traditional care group. Early recovery of normal life, multimodal analgesia and depth of anesthesia monitoring in the ERAS program can effectively reduce surgical stress and the risk of delirium [31]. Opioids are considered to be the cornerstone of analgesics for severe pain, but opioid abuse increases the risk of postoperative delirium [35].

Improved pain control has been proved to be correlated with decreased risks of wound healing and Infectious complications, delirium, delayed mobilization, and prolonged LOS [36, 37]. Multimodal analgesia was applied in our ERAS program, and a standardized analgesic strategy was established based on patient-reported pain VAS score. Significantly lower back pain scores on POD 1-2 and shortened LOS suggested improved pain control in the ERAS program. And nonopioid-preferable pain management can reduce opioid side effects and long-term dependence. It is necessary to weigh the side effects against the strong potency of opioids. Although some guidelines and reviews mentioned about 
Table 7 Multivariable logistic regression for any complications

\begin{tabular}{lll}
\hline Characteristic & Multivariable logistic regression for any complications & \\
\cline { 2 - 3 } & OR $(\mathbf{9 5 \%}$ Cl) & $\boldsymbol{p}$ value \\
\hline Age & $1.02(0.97-1.30)$ & 0.248 \\
BMI & $0.94(0.86-1.21)$ & 0.062 \\
Surgical level $\geq 3$ & $1.87(0.77-4.50)$ & 0.167 \\
ASA $\geq 3$ & $2.06(0.72-5.91)$ & 0.203 \\
Surgical time & $2.23(0.87-3.86)$ & 0.337 \\
ERAS & $0.58(0.19-0.93)$ & 0.040 \\
Preoperative ODI (\%) & $0.91(0.87-1.64)$ & 0.320 \\
\hline
\end{tabular}

multimodal analgesia in ERAS program, there are still some controversies, such as the application of patientspecific multimodal analgesia programs for elderly patients and multimodal analgesic management for preoperative opioid users [12, 35, 38, 39].

Preoperative education helps elderly patients gain a clear understanding of the expectations of surgeries and build confidence in perioperative recovery [40]. Due to the decline in visual and auditory functions of elderly patients, the education was through verbal and handouts, with an emphasis of involvement of family members. Understanding the patient's expectations, preferences and the burden of postoperative care can help medical teams determine better treatment options to truly improve quality of life.

Table 8 Compliance with the ERAS program

\begin{tabular}{ll}
\hline Variable & $\mathbf{n}(\%)$ \\
\hline Pre-operative ERAS items & \\
Patient education & $60(100)$ \\
Nutritional counselling & $60(100)$ \\
No prolonged fasting & $60(100)$ \\
Fluid and carbohydrate loading & $59(98.3)$ \\
Antithrombotic stockings & $58(96.7)$ \\
Antimicrobial prophylaxis & $60(100)$ \\
Intra-operative ERAS items & \\
Tranexamic acid & $60(100)$ \\
Avoidance of salt and water overload & $60(100)$ \\
Maintenance of normothermia & $60(100)$ \\
local infiltration analgesia & $60(100)$ \\
Postoperative ERAS items & \\
Early ambulation & $42(70.0)$ \\
Early removal of bladder catheter & $52(86.7)$ \\
Early oral feeding & $48(80.0)$ \\
Stick to discharge criteria & $47(78.3)$ \\
Perioperative multimodal analgesia & $60(100)$ \\
Overall compliance (rate) & 94.0 \\
\hline
\end{tabular}

A growing number of studies have recognized that malnutrition can lead to adverse outcomes of spinal surgery, especially for elderly patients [41-44]. Increased risk of malnutrition in aging population is due to living alone, chronic diseases and poor dietary habits [45]. Dietitians participated in daily rounds and identified the patients who were malnourished or at risk of malnutrition through nutrition screening tools and laboratory tests. Personalized diet guidance and nutritional supplement were provided to patients in need. Unlike other studies [46], economic factors and medical insurance system were taken into consideration and no additional nutritional supplements were provided for elderly patients with good nutritional status, but instead professional guidance on perioperative diets was given. Our results showed a significant increase in the proportion of patients receiving nutritional supplements in the ERAS group, indicating that previous malnutrition or risks in the non-ERAS traditional care group may be ignored or not intervened.

The neglect of compliance leads to doubts about the impact of ERAS program on the prognosis. Our results illustrated that the overall ERAS compliance was as high as $94 \%$, and the compliance of preoperative and intraoperative items was better than postoperative items. We considered that the postoperative ERAS procedures are affected by the patient's subjective consciousness and the actual condition, while the preoperative and intraoperative steps depend more on the executive capability of the medical team. The close and timely communication of the ERAS team helps to identify potential difficulties and optimize ERAS procedures. Recent studies have shown that continual auditing of the protocol can help to improve compliance [38].

Although the compliance of early ambulation was only $70 \%$ in our study, the overall time until ambulation postoperatively was greatly shortened. Previous studies have shown that early ambulation is associated with decreased morbidity and adverse events after elective lumbar spine surgery [47]. However, preoperative deterioration of motor function, endurance and coordination makes early 
ambulation more difficult. And early ambulation is often accompanied by orthostatic intolerance, such as dizziness and nausea, which increases the risk of aspiration and fall $[47,48]$. Therefore, for elderly patients, early ambulation should be encouraged rather than enforced, and should be accompanied by the presence of professional caregivers and patient confirmation of no obvious discomfort after sitting up.

In this study, $86.7 \%$ of patients complied with early removal of bladder catheter. While early removal of bladder catheter may increase the risk of reinsertion and urinary retention $[30,49]$. It significantly reduces the risk of urinary tract infections and gives patients confidence to return to normal life, which is helpful in shortening LOS [50]. Patient's urination should be closely monitored after early removal of the bladder catheter. Prudence should be taken to determine whether reinsertion is required if there is a possibility of urine retention. Prolonged bladder catheter carrying may be justified for elderly patients with prostate disease.

Although we developed detailed discharge criteria, the compliance was only $78.3 \%$. Geriatric syndromes (such as constipation, incontinence or pressure sores) may cause the elderly not to be discharged even if the discharge criteria are met. In addition, due to inadequate conditions in community medical care facilities and nursing homes, inconvenience of life after discharge and concerns about readmission, some of the elderly patients were reluctant to be discharged $[9,12,51]$. Therefore, we should proactively address the psychosocial problems that the elderly may encounter. Detailed guidance on comorbidities and fostering trust between patients and medical teams can help patients relieve their anxieties. Rehabilitation guidance and telephone follow-up allow patients to be discharged safely.

Individual ERAS components were not independently linked to the prognosis indicators. Nontheless, the ERAS program is a multimodal pathway and all elements have an additive effect on prognosis. The ERAS program is based on "first better, then faster", and extension of LOS in elderly patients with special comorbidities should be granted on a case by case basis.

\section{Limitations}

This was a single-center prospective comparative study with a relatively small sample size. The ERAS and nonERAS traditional care groups were in different time frames, which may cause bias in the analysis. It is not possible to assess the impact of ERAS program on cost savings because no cost information was collected. Due to the short follow-up period, we were unable to evaluate the effects of ERAS program on long-term complications and functional recovery.

\section{Conclusion}

Based on our short-term results, the ERAS program is safer and more effective for elderly patients over 70 undergoing lumbar arthrodesis than non-ERAS traditional care in perioperative period. The ERAS program significantly reduced the incidence of complications and LOS. Due to the characteristics of elderly patients, we should pay attention to compliance when implementing ERAS. For perioperative safety, we should appropriately allow the extension of LOS in elderly patients with special comorbidities.

\section{Abbreviations}

ERAS: Enhanced recovery after surgery; ODI: The Oswestry Disability Index; VAS: Visual analog scales; LOS: Length of stay; ASA: American Society of Anesthesiologists; POD: Postoperative day

\section{Acknowledgements}

We thank the staff at the Department of Orthopedics, Xuanwu Hospital

Capital Medical University, and all the patients who participated in the study.

\section{Authors' contributions}

ZEL was responsible for designing the search strategy, evaluating the articles, running statistical analysis and writing this article. CK was responsible for English editing. SBL and TLW was responsible for designing the protocol. WZS was responsible for interpreting results. PW and STZ were responsible for formatting the paper. All authors have read and approved the final version of the manuscript.

\section{Funding}

This study was sponsored by National Natural Science Foundation of China [No. 81672201 and No. 81871794]. The funders played no role in the design of this study, the collection, analysis and interpretation of data or preparation of the manuscript

\section{Availability of data and materials}

Request for datasets generated and analyzed during the current study can be addressed to the corresponding author.

\section{Declarations}

Ethics approval and consent to participate

This study was reviewed and approved by the institutional review board following the declaration of Helsinki principles in Xuanwu Hospital Capital Medical University. The data collection process was entirely anonymous. The types of questions asked could not bring up any singular information that would make participants identifiable in the database and the results are presented in an aggregate manner. A written informed consent was obtained from all participants of this study.

\section{Consent for publication}

Not applicable.

\section{Competing interests}

The authors declare that they have no competing interests.

\section{Author details}

${ }^{1}$ Department of Orthopedics, Xuanwu Hospital Capital Medical University, Beijing, China. ${ }^{2}$ National Clinical Research Center for Geriatric Diseases, Beijing, China. ${ }^{3}$ Capital Medical University, Beijing, China.

Received: 22 June 2020 Accepted: 11 March 2021

Published online: 17 March 2021

\section{References}

1. Puvanesarajah V, Nourbakhsh A, Hassanzadeh H, Shimer AL, Shen FH, Singla A. Readmission rates, reasons, and risk factors in elderly patients treated 
with lumbar fusion for degenerative pathology. Spine (Phila Pa 1976). 2016; 41:1933-8.

2. Gerhardt J, Bette S, Janssen I, Gempt J, Meyer B, Ryang YM. Is eighty the new sixty? Outcomes and complications after lumbar decompression surgery in elderly patients over 80 years of age. World Neurosurg. 2018;112: e555-60. https://doi.org/10.1016/..wneu.2018.01.082.

3. Rihn JA, Hilibrand AS, Zhao W, Lurie JD, Vaccaro AR, Albert TJ, et al. Effectiveness of surgery for lumbar stenosis and degenerative spondylolisthesis in the octogenarian population: analysis of the spine patient outcomes research trial (SPORT) data. J Bone Jt Surg - Am Vol. 2015; 97(3):177-85. https://doi.org/10.2106/JBJS.N.00313.

4. Kehlet $\mathrm{H}$, Jorgensen CC. Advancing surgical outcomes research and quality improvement within an enhanced recovery program framework. Ann Surg. 2016;264(2):237-8. https://doi.org/10.1097/SLA.0000000000001674.

5. Dagal A, Bellabarba C, Bransford R, Zhang F, Chesnut RM, O'Keefe GE, et al. Enhanced perioperative Care for Major Spine Surgery. Spine (Phila Pa 1976). 2019:44:959-66

6. Kehlet H. Multimodal approach to control postoperative pathophysiology and rehabilitation. Br J Anaesth. 1997;78(5):606-17. https://doi.org/10.1093/ $\mathrm{bja} / 78.5 .606$.

7. Bardram L, Funch-Jensen $P$, Jensen $P$, Kehlet $H$, Crawford ME. Recovery after laparoscopic colonic surgery with epidural analgesia, and early oral nutrition and mobilisation. Lancet. 1995;345(8952):763-4. https://doi.org/10.1016/5014 0-6736(95)90643-6

8. Wang Q, Suo J, Jiang J, Wang C, Zhao YQ, Cao X. Effectiveness of fast-track rehabilitation vs conventional care in laparoscopic colorectal resection for elderly patients: a randomized trial. Colorectal Dis. 2012;14(8):1009-13. https://doi.org/10.1111/j.1463-1318.2011.02855.x.

9. Millan M, Renau-Escrig Al. Minimizing the impact of colorectal surgery in the older patient: the role of enhanced recovery programs in older patients. Eur $J$ Surg Oncol. 2020;46(3):338-43. https://doi.org/10.1016/j.ejso.2019.12.018.

10. Lirosi MC, Tirelli F, Biondi A, Mele MC, Larotonda C, Lorenzon L, et al. Enhanced recovery program for colorectal surgery: a focus on elderly patients over 75 years old. J Gastrointest Surg. 2019;23(3):587-94. https:// doi.org/10.1007/s11605-018-3943-2.

11. Pawa N, Cathcart PL, Arulampalam THA, Tutton MG, Motson RW. Enhanced recovery program following colorectal resection in the elderly patient. World J Surg. 2012;36(2):415-23. https://doi.org/10.1007/s00268-011-1328-8.

12. Kehlet $\mathrm{H}$. Enhanced postoperative recovery: good from afar, but far from good? Anaesthesia. 2020;75:e54-61.

13. Kehlet H, Memtsoudis SG. ERAS guidelines for hip and knee replacementneed for reanalysis of evidence and recommendations? Acta Orthop. 2020: 91(3):243-5. https://doi.org/10.1080/17453674.2020.1728920.

14. Soffin EM, Wetmore DS, Barber LA, Vaishnav AS, Beckman JD, Albert TJ, et al. An enhanced recovery after surgery pathway: association with rapid discharge and minimal complications after anterior cervical spine surgery. Neurosurg Focus. 2019;46:1-9.

15. Feng C, Zhang Y, Chong F, Yang M, Liu C, Liu L, et al. Establishment and implementation of an enhanced recovery after surgery (ERAS) pathway tailored for minimally invasive Transforaminal lumbar Interbody fusion surgery. World Neurosurg. 2019;129:e317-23. https://doi.org/10.1016/j. wneu.2019.05.139.

16. Wang MY, Chang HK, Grossman J. Reduced acute care costs with the ERASR minimally invasive transforaminal lumbar interbody fusion compared with conventional minimally invasive transforaminal lumbar interbody fusion. Clin Neurosurg. 2018;83(4):827-34. https://doi.org/10.1093/neuros/nyx400.

17. Brusko GD, Kolcun JPG, Heger JA, Levi AD, Manzano GR, Madhavan K, et al. Reductions in length of stay, narcotics use, and pain following implementation of an enhanced recovery after surgery program for 1- to 3level lumbar fusion surgery. Neurosurg Focus. 2019;46:1-6.

18. Soffin EM, Vaishnav AS, Wetmore DS, Barber L, Hill P, Gang CH, et al. Design and implementation of an enhanced recovery after surgery (ERAS) program for minimally invasive lumbar decompression spine surgery: initial experience. Spine (Phila Pa 1976). 2019:44:E561-70.

19. von Elm E, Altman DG, Egger M, Pocock SJ, Gøtzsche PC, Vandenbroucke JP. The strengthening the reporting of observational studies in epidemiology (STROBE) statement: guidelines for reporting observational studies. Int J Surg. 2014;12(12):1495-9. https://doi.org/10.1016/j.jpsu.2014.07 013.

20. Ali ZS, Ma TS, Ozturk AK, Malhotra NR, Schuster JM, Marcotte PJ, et al. Preoptimization of spinal surgery patients: development of a neurosurgical enhanced recovery after surgery (ERAS) protocol. Clin Neurol Neurosurg. 2018;164:142-53. https://doi.org/10.1016/j.clineuro.2017.12.003.

21. Lamperti M, Tufegdzic B, Avitsian R. Management of complex spine surgery. Curr Opin Anaesthesiol. 2017;30(5):551-6. https://doi.org/10.1097/ACO. 0000000000000494

22. Wang MY, Green BA, Shah S, Vanni S, Levi ADO. Complications associated with lumbar stenosis surgery in patients older than 75 years of age. Neurosurg Focus. 2003;14(2):1-4. https://doi.org/10.3171/foc.2003.14.2.8.

23. Deyo RA, Mirza SK, Martin BI, Kreuter W, Goodman DC, Jarvik JG. Trends, major medical complications, and charges associated with surgery for lumbar spinal stenosis in older adults. JAMA - J Am Med Assoc. 2010; 303(13):1259-65. https://doi.org/10.1001/jama.2010.338.

24. Røsstad T, Salvesen Ø, Steinsbekk A, Grimsmo A, Sletvold O, Garåsen H. Generic care pathway for elderly patients in need of home care services after discharge from hospital: a cluster randomised controlled trial. BMC Health Serv Res. 2017;17(1):275. https://doi.org/10.1186/s12913-017-2206-3.

25. Webb C, Day R, Velazco CS, Pockaj BA, Gray RJ, Stucky CC, et al. Implementation of an enhanced recovery after surgery (ERAS) program is associated with improved outcomes in patients undergoing Cytoreductive surgery and Hyperthermic Intraperitoneal chemotherapy. Ann Surg Oncol. 2020;27(1):303-12. https://doi.org/10.1245/s10434-019-07900-z.

26. Liu B, Liu S, Wang Y, Zhao L, Zheng T, Chen L, et al. Enhanced recovery after Intraspinal tumor surgery: a single-institutional randomized controlled study. World Neurosurg. 2020;136:e542-52. https://doi.org/10.1016/j.wneu.2 020.01.067.

27. Tejedor P, Pastor C, Gonzalez-Ayora S, Ortega-Lopez M, Guadalajara H, Garcia-Olmo D. Short-term outcomes and benefits of ERAS program in elderly patients undergoing colorectal surgery: a case-matched study compared to conventional care. Int J Colorectal Dis. 2018;33(9):1251-8. https://doi.org/10.1007/s00384-018-3057-z.

28. Shaffer WO, Baisden JL, Fernand R, Matz PG. An evidence-based clinical guideline for antibiotic prophylaxis in spine surgery. Spine J. 2013;13(10): 1387-92. https://doi.org/10.1016/j.spinee.2013.06.030.

29. Kamath VHD, Cheung JPY, Mak KC, Wong YW, Cheung WY, Luk KDK, et al. Antimicrobial prophylaxis to prevent surgical site infection in adolescent idiopathic scoliosis patients undergoing posterior spinal fusion: 2 doses versus antibiotics till drain removal. Eur Spine J. 2016;25(10):3242-8. https:// doi.org/10.1007/s00586-016-4491-7.

30. Okrainec A, Aarts MA, Conn LG, McCluskey S, McKenzie M, Pearsall EA, et al. Compliance with urinary catheter removal guidelines leads to improved outcome in enhanced recovery after surgery patients. J Gastrointest Surg. 2017;21(8):1309-17. https://doi.org/10.1007/s11605-017-3434-x.

31. Petersen PB, Jørgensen CC, Kehlet $H$, Madsen F, Hansen TB, Husted $H$, et al. Delirium after fast-track hip and knee arthroplasty - a cohort study of 6331 elderly patients. Acta Anaesthesiol Scand. 2017;61(7):767-72. https://doi. org/10.1111/aas.12932.

32. Fineberg SJ, Nandyala SV, Marquez-Lara A, Oglesby M, Patel AA, Singh K. Incidence and risk factors for postoperative delirium after lumbar spine surgery. Spine (Phila Pa 1976). 2013:38:1790-6.

33. Pan Z, Huang K, Huang W, Kim KH, Wu H, Yu Y, et al. The risk factors associated with delirium after lumbar spine surgery in elderly patients. Quant Imaging Med Surg. 2019;9(4):700-10. https://doi.org/10.21037/qims.2 019.04.09.

34. Krenk $L$, Rasmussen $L S$, Kehlet $H$. New insights into the pathophysiology of postoperative cognitive dysfunction. Acta Anaesthesiol Scand. 2010;54(8): 951-6. https://doi.org/10.1111/j.1399-6576.2010.02268.x.

35. Cozowicz C, Bekeris J, Poeran J, Zubizarreta N, Schwenk E, Girardi F, et al. Multimodal pain management and postoperative outcomes in lumbar spine fusion surgery: a population-based cohort study. Spine (Phila Pa 1976). 2020; 45:580-9.

36. Dietz N, Sharma M, Adams S, Alhourani A, Ugiliweneza B, Wang D, et al. Enhanced recovery after surgery (ERAS) for spine surgery: a systematic review. World Neurosurg. 2019;130:415-26. https://doi.org/10.1016/j.wneu.2 019.06.181.

37. Alboog A, Bae S, Chui J. Anesthetic management of complex spine surgery in adult patients: a review based on outcome evidence. Curr Opin Anaesthesiol. 2019;32(5):600-8. https://doi.org/10.1097/ACO. 0000000000000765 .

38. Schwartz AR, Lim S, Broadwater G, Cobb L, Valea F, Marosky Thacker J, et al. Reduction in opioid use and postoperative pain scores after elective laparotomy with implementation of enhanced recovery after surgery 
protocol on a gynecologic oncology service. Int J Gynecol Cancer. 2019; 29(5):935-43. https://doi.org/10.1136/ijgc-2018-000131.

39. Joshi GP, Kehlet H, Beloeil H, Bonnet F, Fischer B, Hill A, et al. Guidelines for perioperative pain management: need for re-evaluation. Br J Anaesth. 2017; 119(4):720-2. https://doi.org/10.1093/bja/aex304.

40. Eastwood D, Manson N, Bigney E, Darling M, Richardson E, Paixao R, et al. Improving postoperative patient reported benefits and satisfaction following spinal fusion with a single preoperative education session. Spine J. 2019;19(5):840-5. https://doi.org/10.1016/j.spinee.2018.11.010.

41. Tempel Z, Grandhi R, Maserati M, Panczykowski D, Ochoa J, Russavage J, et al. Prealbumin as a serum biomarker of impaired perioperative nutritional status and risk for surgical site infection after spine surgery. J Neurol Surgery, Part A Cent Eur Neurosurg. 2015;76:139-43.

42. Qureshi R, Rasool M, Puvanesarajah V, Hassanzadeh H. Perioperative nutritional optimization in spine surgery. Clin Spine Surg. 2018;31(3):103-7. https://doi.org/10.1097/BSD.0000000000000579.

43. Ostrowska J, Szostak-Węgierek D, Jeznach-Steinhagen A. Nutritional status in patients aged over 65 years and its influence on the quantity and type of complications occurring 3, 6 and 12 months after hospitalisation: a clinical prospective study. J Hum Nutr Diet. 2019;32(1):119-27. https://doi.org/1 $0.1111 /$ jhn. 12590 .

44. Ukogu CO, Jacobs S, Ranson WA, Somani S, Vargas L, Lee NJ, et al. Preoperative nutritional status as a risk factor for major postoperative complications following anterior lumbar Interbody fusion. Glob Spine J. 2018;8(7):662-7. https://doi.org/10.1177/2192568218760540.

45. Dos Santos Junqueira JC, Cotrim Soares E, Rodriques Corrêa Filho H, Fenalti Hoehr N, Oliveira Magro D, Ueno M. Nutritional risk factors for postoperative complications in Brazilian elderly patients undergoing major elective surgery. Nutrition. 2003;19(4):321-6. https://doi.org/10.1016/50899-9007(02 )00863-8.

46. Xu B, Xu WX, Lao YJ, Ding WG, Lu D, Sheng HF. Multimodal nutritional Management in Primary Lumbar Spine Surgery: a randomized controlled trial. Spine (Phila Pa 1976). 2019;44:967-74

47. Zakaria HM, Bazydlo M, Schultz L, Abdulhak M, Nerenz DR, Chang V, et al. Ambulation on postoperative Day \#0 is associated with decreased morbidity and adverse events after elective lumbar spine surgery: analysis from the Michigan spine surgery improvement collaborative (MSSIC). Neurosurgery. 2020;87(2):320-8. https://doi.org/10.1093/neuros/nyz501.

48. Jans $\varnothing$, Kehlet H. L'intolérance orthostatique postopératoire: malgré un problème périopératoire fréquent, peu de solutions. Can J Anesth. 2017; 64(1):10-5. https://doi.org/10.1007/s12630-016-0734-7.

49. Kwaan MR, Lee JT, Rothenberger DA, Melton GB, Madoff RD. Early removal of urinary catheters after rectal surgery is associated with increased urinary retention. Dis Colon Rectum. 2015;58(4):401-5. https://doi.org/10.1097/DCR. 0000000000000317.

50. Eriksen JR, Munk-Madsen P, Kehlet H, Gögenur I. Postoperative urinary retention after laparoscopic colorectal resection with early catheter removal: a prospective observational study. World J Surg. 2019;43(8):2090-8. https:// doi.org/10.1007/s00268-019-05010-1.

51. Lu SY, Lai Y, Dalia AA. Implementing a cardiac enhanced recovery after surgery protocol: nuts and bolts. J Cardiothorac Vasc Anesth. 2020;34(11): 3104-12. https://doi.org/10.1053/j.jvca.2019.12.022.

\section{Publisher's Note}

Springer Nature remains neutral with regard to jurisdictional claims in published maps and institutional affiliations.

Ready to submit your research? Choose BMC and benefit from:

- fast, convenient online submission

- thorough peer review by experienced researchers in your field

- rapid publication on acceptance

- support for research data, including large and complex data types

- gold Open Access which fosters wider collaboration and increased citations

- maximum visibility for your research: over $100 \mathrm{M}$ website views per year

At BMC, research is always in progress.

Learn more biomedcentral.com/submissions 